\title{
Quantification of piscine reovirus (PRV) at different stages of Atlantic salmon Salmo salar production
}

\author{
Marie Løvoll, Marta Alarcón, Britt Bang Jensen, Torunn Taksdal, \\ Anja B. Kristoffersen, Torstein Tengs*
}

Norwegian Veterinary Institute, Ullevaalsveien 68, 0454 Oslo, Norway

\begin{abstract}
The newly described piscine reovirus (PRV) appears to be associated with the development of heart and skeletal muscle inflammation (HSMI) in farmed Atlantic salmon Salmo salar L. PRV seems to be ubiquitous among fish in Norwegian salmon farms, but high viral loads and tissue distribution support a causal relationship between virus and disease. In order to improve understanding of the distribution of PRV in the salmon production line, we quantified PRV by using realtime PCR on heart samples collected at different points in the life cycle from pre-smolts to fish ready for slaughter. PRV positive pre-smolts were found in about $36 \%$ of the freshwater cohorts and a general increase in viral load was observed after their transfer to seawater. A reduction in viral loads was recorded when fish approached slaughter (18 mo in sea cages). Sequencing of positive samples did not support the hypothesis that outbreaks are caused by the spreading of a particular (virulent) strain of PRV.
\end{abstract}

KEY WORDS: Piscine reovirus $\cdot$ PCR $\cdot$ Salmon $\cdot$ Aquaculture $\cdot$ HSMI

\section{INTRODUCTION}

Heart and skeletal muscle inflammation (HSMI) is one of the most common diseases in farmed Atlantic salmon Salmo salar L. in Norway. The number of outbreaks of HSMI has increased steadily since the first report in 1999 (Kongtorp et al. 2004a,b). In 2010, 131 farms in Norway had reports of the disease. In certain regions, HSMI was diagnosed at more than $90 \%$ of the farms at least once during 2010 (Farmed Fish Health Report 2010, www.vetinst.no/eng/content/ download/8034/98977/file/Fish_Health_Report_2010. pdf). Mortality from HSMI is generally low to moderate, but cumulative mortalities of up to $20 \%$ have been reported (Kongtorp et al. 2006).

Clinical disease is usually discovered when reduced appetite, abnormal swimming behaviour, moribund fish and/or increased mortality is observed in one or more cages. Often, stressors such as moving the fish or treatments for sea lice have preceded the outbreaks. As determined by necropsy the most com- mon findings are hemopericardium and/or a pale or grayish heart, as well as signs of circulatory disturbance such as ascitic fluid, swollen spleen and/or accumulation of blood in tissues. A fibrinous coat on the liver surface may be found. However, neither clinical signs nor macroscopic changes are specific for HSMI. Therefore, the disease is currently diagnosed by detection of characteristic histopathology (Kongtorp et al. 2004b).

Usually, the disease occurs from 5 to 9 mo after transfer of the salmon from freshwater tanks to seawater cages, but outbreaks have also been reported as early as $2 \mathrm{wk}$ after exposure to seawater (Kongtorp et al. 2004b). Cardiac lesions consistent with HSMI have been detected in fish both before and after clinical outbreaks and also in samples collected from clinically healthy fish (Kongtorp et al. 2006). Based on the history of HSMI outbreaks, it has been assumed that freshwater stages of Atlantic salmon are not affected (Kongtorp et al. 2006). 
After initial success with experimental transmission of the disease (Kongtorp et al. 2004a), HSMI was recently linked to a novel reovirus, piscine reovirus (PRV) (Palacios et al. 2010). Although the virus appears to be ubiquitous in Norwegian salmon farms, elevated viral loads are associated with outbreaks of HSMI (Palacios et al. 2010). A causal relationship is also supported by in situ hybridisation using formalin-fixed heart tissue where a correlation between the presence of viral nucleic acids and histopathological lesions has been found (Palacios et al. 2010). PRV has been detected in healthy, wild salmon, albeit with lower prevalence and virus load. The virus has also been found in archive samples taken from salmon in the late 1980s and also in rainbow trout Oncorhynchus mykiss Walbaum (Palacios et al. 2010). Farmed Atlantic salmon with HSMI-like lesions have also been reported from Scotland (Ferguson et al. 2005).

In the present study we quantified PRV loads at various stages in the Atlantic salmon production line, from pre-smolts in fresh water to fish ready for slaughter. Samples were collected from farms where no heart lesions were observed and from farms with clinical outbreaks of HSMI. We also sequenced parts of the PRV genome detected in different samples in order to investigate the molecular epidemiology of the virus and potentially virulent PRV strains. Our results indicate that PRV is a ubiquitous virus in Norwegian fish farms and that careful virus quantification and/or study of tissue distribution of viral particles/genomes need to be complemented with histopathology for correct diagnoses. The results should be useful when interpreting PRV loads both in the context of HSMI/PRV epidemiology and diagnosis.

\section{MATERIALS AND METHODS}

Viral loads were established for 2 sets of samples; one set of 171 field samples was collected from 23 marine fish farms and sent to the Norwegian Veterinary Institute (NVI) for disease diagnostics during 2010, and 460 samples were collected from a longitudinal study. The latter sample series included samples from pre-smolts (in fresh water), smolts 2 and 8 mo after seawater transfer and samples collected just prior to slaughter (10 fish in each set; 14 farms). For more details about this sample set, see Jansen et al. (2010).

Total nucleic acids were extracted from homogenates of ventricular heart tissue, which had been stored in RNAlater (Qiagen $A B$ ), by using the
NucliSENS easyMAG nucleic acid extraction system (bioMérieux) according to the manufacturer's recommendations. Specimens were transferred from RNAlater to NucliSens lysis buffer (bioMérieux) and homogenised with a mixer mill homogeniser (model MM 400, Retsch Norge AS) and 3 mm tungsten carbide beads (Qiagen). RNA yields were quantified with a spectrophotometer (Thermo Scientific NanoDrop 2000, NanoDrop Products).

Viral quantification by real-time reverse transcription quantitative polymerase chain reaction (RTqPCR) was done using the OneStep RT-PCR kit (Qiagen) and forward primer TGC TAA CAC TCC AGG AGT CAT TG, reverse primer TGA ATC CGC TGC AGA TGA GTA and MGB probe CGC CGG TAG CTC T (all written 5'-3') targeting the L1 fragment of the PRV genome. The MGB probe (Applied Biosystems) was labelled at the 5' end with 6-FAM and at the 3' end with minor groove binder/nonfluorescent quencher. Primer and probes were identical to those used by Palacios et al. (2010). The final concentrations of primers and probe were 400 and $240 \mathrm{nM}$, respectively. A $100 \mathrm{ng}$ sample of RNA was used as template, and ROX (Roche Diagnostics Norge AS) was included as a passive reference dye. The following thermal cycling parameters were used: $30 \mathrm{~min}$ at $50^{\circ} \mathrm{C}$ (reverse transcription), $15 \mathrm{~min}$ at $95^{\circ} \mathrm{C}$ (inactivation of reverse transcriptase and activation of hotstart PCR DNA polymerase), $30 \mathrm{~s}$ at $94^{\circ} \mathrm{C}$ (template denaturation) and $60 \mathrm{~s}$ at $60^{\circ} \mathrm{C}$ (primer annealing; 45 cycles total). Threshold values were set using default settings and threshold cycle $\left(\mathrm{Ct}_{\text {; }} \mathrm{PCR}\right.$ cycle number where the signal from a sample is defined as positive by the software) scores were established using the dRn formula (baseline subtracted fluorescence reading normalized to the reference dye). All RT-qPCR analyses were performed using the Mx3000P QPCR System (Agilent Technologies).

Based on preliminary sequence data using PRV material isolated from different outbreaks and locations we determined that the S4 segment of the viral genome shows the highest degree of variability (both coding and non-coding changes; data not shown). Among our PRV positive samples, a subset representing cohorts with different HSMI histories and presence or absence of PRV positive pre-smolts were selected for sequencing. Near full-length S4 (1017 bp) was PCR-amplified using 2 overlapping sets of primers: (1) S4_39F AAA CCC AAA TGG CGA ACC A and S4_621R GAG CTG AAC CCA GTG GAG CA; (2) S4_460F TTG AAG CTA AGC GAC GCC TT and S4_1036R CGT GAT GGG GAG CCT ACT GT (5'-3'). The OneStep RT-PCR kit (QIA- 
GEN) was used with final primer concentrations of $600 \mathrm{nM}$ and the following PCR cycle: $30 \mathrm{~min}$ at $50^{\circ} \mathrm{C}$ (reverse transcription), $15 \mathrm{~min}$ at $95^{\circ} \mathrm{C}$ (inactivation of reverse transcriptase and activation of hot-start PCR DNA polymerase), $30 \mathrm{~s}$ at $94^{\circ} \mathrm{C}$ (template denaturation), $30 \mathrm{~s}$ at $55^{\circ} \mathrm{C}$ (primer annealing, $1 \mathrm{~min}$ at $72^{\circ} \mathrm{C}$ (primer elongation; 45 cycles total) and a final elongation step $\left(3 \mathrm{~min}\right.$ at $72^{\circ} \mathrm{C}$ ). All amplification reactions gave a single, clear band when analysed with a 2100 Bioanalyzer (Agilent Technologies) according to the manufacturer's recommendations. Unincorporated primers and nucleotides were removed from the PCR products by using ExoSAP-IT (GE Healthcare) and the products were sequenced using Sanger sequencing and the PCR primers.

$\mathrm{Ct}$ values from the diagnostic samples were visualized through a box plot showing the median and the quartiles of the samples from farms with HSMI outbreaks compared with farms without disease. A mixed-effect regression model with months since seawater transfer and presence or absence of HSMI as a fixed effect and location as random effect was used to analyse the virus quantification data and to verify that the differences in $\mathrm{Ct}$ values were real and not due to either time in sea cages or different samplings areas.

$\mathrm{Ct}$ values for all 4 time points from the longitudinal study were visualised by using a box plot of the samples from cohorts with reports of HSMI and compared with cohorts without reports of the disease. Ten fish were analysed per cohort per time point, $\mathrm{Ct}$ values above 40 were defined as negative (noise/low level contamination) and negative RT-qPCRs were set to $\mathrm{Ct}=45$. Farms were sampled according to time points, and HSMI or cardiomyopathy syndrome (CMS) diagnoses were thus set independent of these samplings.

HSMI and CMS were diagnosed on farm or cohort level (not using the individuals included in this study) by means of histopathological evaluations of multiple specimens from several individuals. Sections of formalin-fixed, paraffin-embedded, hematoxylin and eosin (H\&E) stained hearts were examined with light microscopy. An HSMI diagnosis requires the presence of severe inflammatory infiltrates in the epicardium and in the compact and spongious muscle layers of the heart ventricle. Other organs were also examined in order to secure differentiation from other diseases. All diagnoses were made by experienced pathologists at the NVI with broad experience in diagnostic work, research, or both, on heart diseases in Atlantic salmon. Histopathological evaluations of PRV-positive pre-smolts were done on the same individuals as used for RNA extraction and RTqPCR.

\section{RESULTS}

When comparing viral loads from individual fish collected from marine farms without HSMI $(n=109)$ with fish sampled during HSMI outbreaks ( $\mathrm{n}=62$ ), a clear shift in Ct values could be seen (Fig. 1). Mixedeffect regression showed that the number of months in seawater was not a significant variable $(\mathrm{p}=0.23)$ and that the shift between the groups was significant $(p<0.001)$. A logistic regression plot of the data revealed that a potential cutoff value for setting an HSMI diagnosis would be approximately 28 (Fig. S1 in the Supplement at www.int-res.com/articles/supp/ d099p007_supp.pdf). Nineteen fish from farms without HSMI tested negative with the RT-qPCR assay whereas only 1 fish from an HSMI outbreak tested negative.

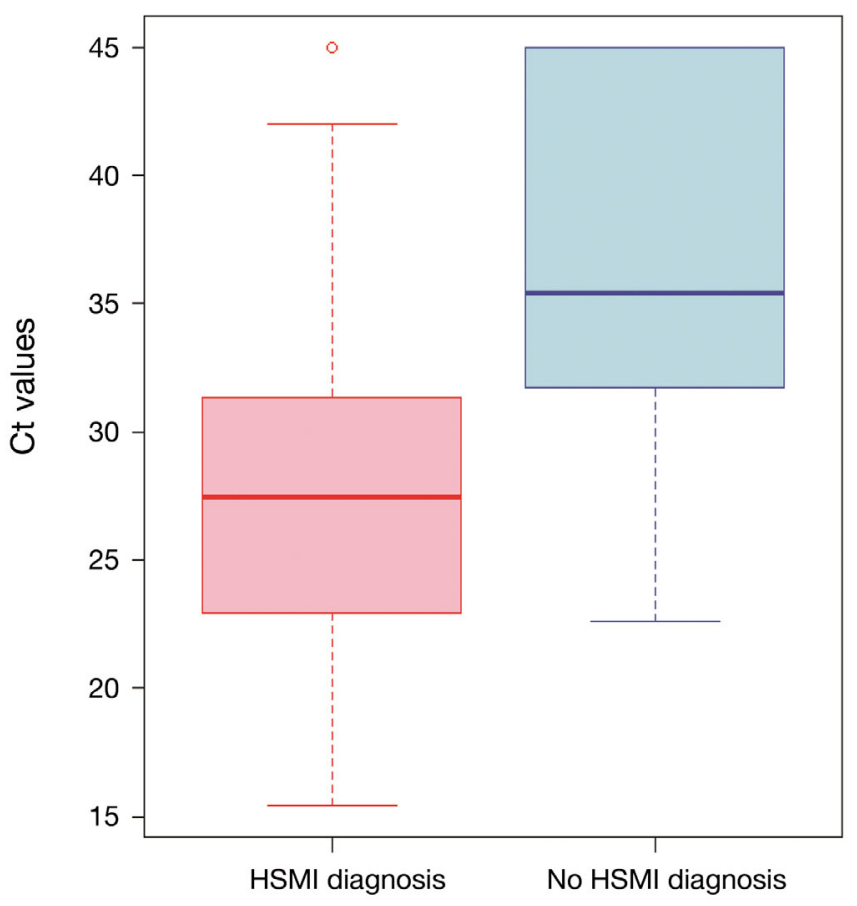

Fig. 1. Box plot of Ct values (= 45 for negative RT-qPCRs) for farms that did not experience heart and skeletal muscle inflammation (HSMI; $\mathrm{n}=109$ ) in Atlantic salmon and farms at which HSMI was diagnosed $(\mathrm{n}=62)$. Thick lines show the median, and boxes reflect quartiles. Ends of whiskers represent the lowest $\mathrm{Ct}$ value still within the 1.5 interquartile range (IQR) of the lower quartile and the highest $\mathrm{Ct}$ value still within the 1.5 IQR of the upper quartile; open circle indicates an outlier. Diagnosis was made at the farm level and was not based on the same individual fish as the samples used for RT-qPCR 
None of the cohorts remained PRV-free throughout the test period. Some of the hatcheries had presmolts that tested positive for PRV (5 of 14) and some of these fish appeared to have relatively high viral loads (Ct values < 25; Table S1). A general increase in PRV loads or prevalence after transfer to seawater was observed both for cohorts with subsequent reports of HSMI and for cohorts that did not experience HSMI outbreaks at any time during the seawater production (Fig. 2). Six farms reported outbreaks of HSMI during the sampling period, and the highest viral loads could be seen in samples collected at these sites at 8 mo (sampling time point closest to time of diagnosis; Fig. 2, Table S1). There was also a consistent decrease in viral load and/or number of positive samples when comparing samples taken 8 mo after sea transfer with samples from fish ready for slaughter, both for cohorts with HSMI history and cohorts in the 'no heart lesions' group (Fig. 2, Table S1). The longitudinal samples were not collected specifically for HSMI screening, and thus PRV loads were not established for the peak phase of the HSMI outbreaks and diagnoses were made by means of histopathological examination of samples collected during the outbreak events independent of the sampling for RT-qPCR.

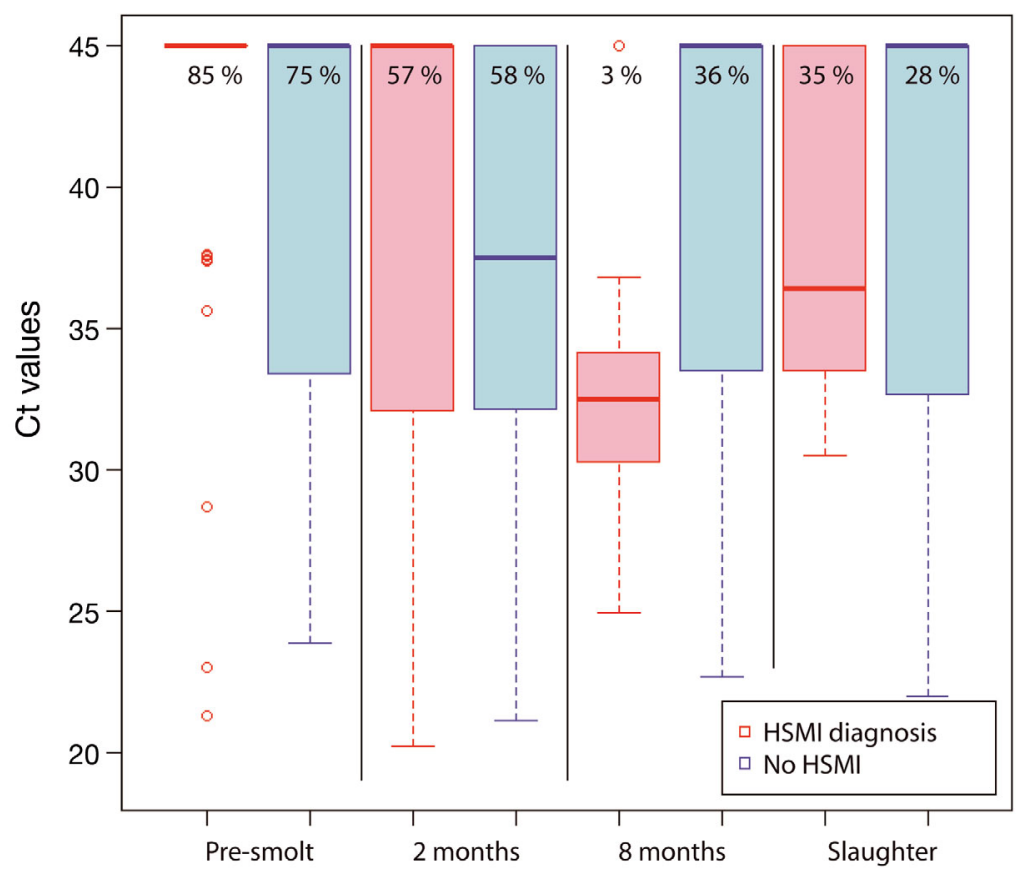

Fig. 2. Box plot of $\mathrm{Ct}$ values at multiple time points in the production cycle for Atlantic salmon cohorts with $(n=6$, red) and without $(n=8$, blue) occurrence of HSMI. Box plot details are as in Fig. 1; open circles indicate outliers. The percentage of RT-qPCRs that were negative is indicated for each group
A 1017 bp S4 genome fragment was sequenced from 27 samples collected both from outbreak and 'no lesions' cohorts. The sequences (submitted to GenBank as accessions JN991006 to JN991012) revealed that there were no shared genetic features distinguishing the outbreak cohorts from the cohorts with no history of HSMI (Fig. S2). Also, apart from a small number of silent/conservative single nucleotide polymorphisms (SNPs), no differences were found when samples representing the same cohorts were compared. For Cohort 1, a single SNP was found when the S4 sequence from a pre-smolt was compared with other samples (other pre-smolts and later time points) and 2 samples collected at Week 8 had a single SNP when Cohorts 9 and 6 were examined (Fig. S2).

\section{DISCUSSION}

In the present study, farmed Atlantic salmon were screened for presence of PRV throughout the production cycle, from pre-smolts to harvest. The results confirmed the general assumption that PRV is a ubiquitous virus in Norwegian salmon production farms. Our results also support the hypothesis that a significant increase in viral load is associated with the development of HSMI (Palacios et al. 2010).

Some of the cohorts showed the presence of PRV in salmon pre-smolts. Although the majority of freshwater farms were RT-qPCR negative (9 of 14), the possibility of vertical transmission of the virus cannot be excluded if the broodfish were carriers of PRV at the time of egg stripping. However, it was recently shown that eggs from PRVpositive broodfish were PRV-negative under commercial hatching conditions (Wiik-Nielsen et al. 2012). PRV infection of smolts may also occur by horizontal transmission of PRV through seawater. Some hatcheries introduce seawater into their smolt production to reduce freshwater costs or increase $\mathrm{pH}$ or, in some cases, as an attempt to prevent haemorrhagic smolt syndrome. Based on the high prevalence of PRV in farmed salmon in seawater, horizontal spread of the virus between field locations represents a likely route of transmission. Indeed, a study performed by Aldrin et 
al. (2010) demonstrated that a model using horizontal transmission of HSMI explained the disease pattern well, and the risk of disease increased as distance to a neighboring infected farm decreased. Similar to infectious pancreatic necrosis virus (IPNV), PRV is a naked virus containing double-stranded RNA. These viruses are known to be robust and resistant to adverse physical conditions and disinfection, and there will be an increased risk of transmission of these viruses through inadequate disinfection of pipelines and equipment.

As with other fish viruses, such as infectious salmon anemia virus (ISAV; Mjaaland et al. 2002, Markussen et al. 2008) and IPNV (Song et al. 2005), it has been speculated that sequence variation in the PRV genome may account for strains with different virulence. To investigate this, PRV segment S4 was chosen for sequencing. Based on in silico analyses, it was determined that S4 was likely to encode the sigma $3(\sigma 3)$ gene product that resides on the outer surface of the virus particle (T. Markussen, pers. comm.). Sigma 3 can be relevant for virulence in other reoviruses (Yue \& Shatkin 1997) and thus should be a good candidate for frequent mutations and rearrangements in PRV. When comparing the S4 sequences generated from fish sampled during HSMI outbreaks with sequence data from fish from cohorts that had no history of HSMI and no heart lesions, no consistent amino acid or nucleotide differences were observed. In addition, the clusters observed in the phylogenetic analysis (Fig. S2) did not reveal any patterns corresponding to any identifiable variable, such as geographic origin of samples for instance. The level of sequence diversity observed is comparable with what has previously been reported from populations of PRV (Løvoll et al. 2010). Absence of genetic features defining PRV strains involved in outbreaks makes it unlikely that HSMI is caused by the spreading of a specific, virulent strain of PRV. If there are genetically determined virulent strains of the virus, our data suggest that such quasispecies develop de novo in association with outbreaks and that any such virulence markers reside on genome fragments other than S4.

No consistent histopathological lesions could be seen in the pre-smolts with high loads of PRV. The possibility of biased quantification cannot be ruled out when it comes to size of heart tested or developmental stage of fish. In the original paper describing PRV and its correlation with HSMI, a reference gene was used for normalization of PRV RT-qPCR results (Palacios et al. 2010). Based on data from a large number of different samples this particular approach to PRV quantification was found to be very sensitive to expression levels of the reference gene and to differences in the integrity of cellular versus viral RNA (T. Tengs unpubl. data). Methods for PCR-based viral quantification all have their limitations. Based on extensive testing we have decided to use standard input amount of total RNA for relative quantification. This method has also been implemented for routine PRV screening of farmed salmon by the NVI.

Regardless of the exact viral loads, PRV positive pre-smolts were detected both in cohorts that remained disease free and in cohorts that experienced HSMI at later stages, indicating that early infection probably does not induce an effective immunizing effect. Assuming there is an equal degree of virulence for the strains of PRV detected, it is possible that the immune system of pre-smolts is not sufficiently developed to give a strong cellular immune response. Tolerance towards the virus may allow pre-smolts to harbour high loads of PRV without developing a strong inflammatory reaction. These observations are compatible with a model where environmental factors associated with the seawater locations are more important than PRV status in presmolts, as early exposure to virus and high viral loads do not always result in adult fish developing HSMI.

In the present study, fish in one farm were diagnosed with CMS 1 yr after an outbreak of HSMI (Table S1). It has been shown previously that PRV and the causative agent of CMS, piscine myocarditis virus (PMCV), can establish a co-infection (Løvoll et al. 2010). Whether there is a synergistic effect of coinfection or a predisposition for CMS after an infection with PRV remains to be investigated.

Considering the ubiquitous nature and persistence of PRV, it is not likely that vaccination alone will eradicate the virus from the aquaculture industry. But, as with other prophylactic measures, vaccination may contribute to reducing the number and/or severity of HSMI outbreaks. The decrease in viral loads seen in fish ready for slaughter may indicate that the fish are able to mount a certain immunological response against PRV. Selective breeding for resistance is an additional strategy for fighting HSMI. Genetically improved resistance in Atlantic salmon populations has been a helpful addition to vaccination against infectious pancreatic necrosis, which causes major economic losses in fry and post-smolts (Storset et al. 2007). So far, there is little documentation available on the genetic variation in resistance to HSMI in a breeding population, but it is possible that selective breeding may contribute to controlling the disease. 
Since PRV was identified in 2010, a lot of data has been generated from analysis of diagnostic samples linked to outbreaks of HSMI. The present work adds to the general assumption that PRV is a ubiquitous virus and also confirms that elevated PRV loads are associated with HSMI outbreaks. Additionally, the data indicate that the fish are capable of reducing, but not clearing, the viral load by the end of the production cycle.

Acknowledgements. This work was funded by the Norwegian Veterinary Institute (project 2011022) and The Research Council of Norway (SIP-FKD project number 186907). The authors thank G. Bornø, O. Bendik Dale, C. Fritsvold, M. Gjessing, H. Hellberg, R. Johansen, H. R. Skjelstad and C. Sviland for help with sample collection and E. Rimstad for critical reading of the manuscript.

\section{LITERATURE CITED}

Aldrin M, Storvik B, Frigessi A, Viljugrein H, Jansen PA (2010) A stochastic model for the assessment of the transmission pathways of heart and skeleton muscle inflammation, pancreas disease and infectious salmon anaemia in marine fish farms in Norway. Prev Vet Med 93:51-61

Ferguson HW, Kongtorp RT, Taksdal T, Graham D, Falk K (2005) An outbreak of disease resembling heart and skeletal muscle inflammation in Scottish farmed salmon, Salmo salar L., with observations on myocardial regeneration. J Fish Dis 28:119-123

Jansen MD, Taksdal T, Wasmuth MA, Gjerset B and others (2010) Salmonid alphavirus (SAV) and pancreas disease (PD) in Atlantic salmon, Salmo salar L., in freshwater and seawater sites in Norway from 2006 to 2008. J Fish Dis 33:391-402

Kongtorp RT, Kjerstad A, Taksdal T, Guttvik A, Falk K (2004a) Heart and skeletal muscle inflammation in Atlantic salmon, Salmo salar L.: a new infectious disease. J Fish Dis 27:351-358

Editorial responsibility: Mark Crane,

Geelong, Victoria, Australia
Kongtorp RT, Taksdal T, Lyngoy A (2004b) Pathology of heart and skeletal muscle inflammation (HSMI) in farmed Atlantic salmon Salmo salar. Dis Aquat Org 59: $217-224$

> Kongtorp RT, Halse M, Taksdal T, Falk K (2006) Longitudinal study of a natural outbreak of heart and skeletal muscle inflammation in Atlantic salmon, Salmo salar L. J Fish Dis 29:233-244

> Løvoll M, Wiik-Nielsen J, Grove S, Wiik-Nielsen CR and others (2010) A novel totivirus and piscine reovirus (PRV) in Atlantic salmon (Salmo salar) with cardiomyopathy syndrome (CMS). Virol J 7:309

Markussen T, Jonassen CM, Numanovic S, Braaen S, Hjortaas M, Nilsen H, Mjaaland S (2008) Evolutionary mechanisms involved in the virulence of infectious salmon anaemia virus (ISAV), a piscine orthomyxovirus. Virology 374:515-527

> Mjaaland S, Hungnes O, Teig A, Dannevig BH, Thorud K, Rimstad E (2002) Polymorphism in the infectious salmon anemia virus hemagglutinin gene: importance and possible implications for evolution and ecology of infectious salmon anemia disease. Virology 304:379-391

- Palacios G, Lovoll M, Tengs T, Hornig M and others (2010) Heart and skeletal muscle inflammation of farmed salmon is associated with infection with a novel reovirus. PLoS One 5:e11487

> Song H, Santi N, Evensen O, Vakharia VN (2005) Molecular determinants of infectious pancreatic necrosis virus virulence and cell culture adaptation. J Virol 79: 10289-10299

> Storset A, Strand C, Wetten M, Sissel K, Rarnstad A (2007) Response to selection for resistance against infectious pancreatic necrosis in Atlantic salmon (Salmo salar L.). Aquaculture 272:S62-S68

> Wiik-Nielsen CR, Ski PMR, Aunsmo A, Løvoll M (2012) Prevalence of viral RNA from piscine reovirus (PRV) and piscine myocarditis virus (PMCV) in Atlantic salmon, Salmo salar L., broodfish and progeny. J Fish Dis 35: 169-171

> Yue Z, Shatkin AJ (1997) Double-stranded RNA-dependent protein kinase (PKR) is regulated by reovirus structural proteins. Virology 234:364-371

Submitted: November 7, 2011; Accepted: February 2, 2012 Proofs received from author(s): May 7, 2012 